\title{
Parasitism and environmental pollution: parasites and hosts as indicators of water quality
}

\author{
Edited by JOHN LEWIS and DAVID HOOLE \\ CO-ORDINATING EDITOR L. H. CHAPPELL
}

\section{Preface}

Parasitic organisms are found in almost every environment ranging from the extremes of cold conditions in the polar regions to hot climates in the tropics. Natural abiotic factors such as temperature, oxygen, salinity and hydrogen ion concentration are known to influence the temporal and spatial occurrence of parasites and in particular the helminths of fish (Chubb, 1979, 1980). Several scientists through their efforts to use fish parasites as biological tags to assess fish populations have appreciated the importance of parasites as sensitive probes to monitor a range of environmental factors including stress due to pollution (Mackenzie et al. 1995; Lafferty, 1997). Fish parasites are also efficient monitors of the physiological-immunological state of their fish hosts. The main pathways used by assemblages of aquatic parasites in response to pollutants were reviewed by Poulin (1992) who showed that parasite communities are influenced indirectly by pollutants which are toxic both to fish and invertebrate hosts and directly to the parasites and their free living stages. Whilst fish and invertebrates have been used extensively in standard toxicity testing, Kennedy (1997) has argued that the relationships between the environment and the fish host and its parasites will not provide an easy option for monitoring environmental change in pollution incidents. Kennedy also suggested that parasites of aquatic hosts and, in particular, fish did not offer clear advantages over the use of free living organisms as indicators of pollution other than providing additional, or confirmatory, sources of information. However much progress has been made over the past five years and papers presented at the 2002 Autumn Symposium demonstrate how aquatic hosts and their parasites are likely to indicate changes in water quality and play a significant role in our understanding of natural aquatic ecosystems.

The topics in this volume consider the impact of pollution on host-parasite systems in the marine and freshwater environment, commencing with Morley $\&$ Irwin who, in their presentation on the effects of pollution on the transmission of larval digeneans through molluscan hosts, show that complex interactions influence parasite populations under extreme environmental conditions. To date, laboratory experiments, especially with heavy metals, have to a large extent demonstrated those toxicity effects which occur as episodic pollution events. Apart from standardising experimental procedures, more combined laboratory and field studies are needed to clarify the complexity of abiotic and biotic factors involved in the transmission of digeneans under natural conditions. In the marine environment, Williams \& Mackenzie provide an update on criteria used for selecting marine parasites as indicator/ monitor species and data on the monogenean species Diclidophora merlangi and Dictyocotyle coeliaca confirm their value as potential indicators of hydrocarbon pollution in the North Sea. In their study on pollution and parasites in Finnish Lakes, Valtonen et al. report that some parasite communities in fish show evidence of recovery following a reduction in chemical and nutrient loading from a pulp mill in 
central Finland. Apart from an increase in the population of anadontid clams, enhanced immune responses in the fish also reflect much improvement in water quality.

The paper by Sures summarises our present knowledge on parasites as bioindicators and demonstrates from field and laboratory studies that acanthocephalans in particular have the capacity for accumulating heavy metals, the concentrations of which significantly exceed those in the fish tissues. Acanthocephalans can also respond very rapidly to changes in environmental exposure and are more reliable indicators of water quality than their fish hosts.

This is confirmed by Konecny et al. with studies on heavy metal accumulation by the acanthocephalan Pomphorhynchus laevis occurring in the barbel from river sites in Austria. Furthermore, changes in the diversity and richness of endoparasites in the barbel are related to levels of heavy metals in the water but further investigations on food web dynamics and intermediate host populations in these river stretches are needed before precise conclusions can be drawn.

At the cellular level, the presentation by Hoole \& Lewis focuses on inflammatory interactions in carp exposed to pollution and the blood parasite Sanguinicola inermis. The nature of the pollutant and the sequence in which carp is exposed to parasite and pollutant influences the ultrastructural appearance and cellular content of the pronephros and thymus in the fish host. An increase in apoptosis, induced by cortisol, in the pronephric cells of carp is associated with an increase in phagocytosis. The role of apoptosis in the pronephros and $\mathrm{C}$ reactive protein isolated from the serum of carp on inflammatory responses in the fish is discussed and preliminary evidence suggests that levels of $\mathrm{C}$ reactive protein, which is used as a biomarker for inflammatory responses in humans, and other pentraxins may increase during trauma. In their paper on proliferative kidney disease (PKD), Secombes et al. examine the expression of a range of trout cytokines and cytokine-related genes in naïve unexposed fish and in naive fish exposed to $\mathrm{PKD}$-infected water and sampled during the course of a natural outbreak of PKD. Specific immune responses at the molecular level are affected during a PKD infection and cortisol suppression of cytokines expressed in vitro provides a possible link to PKD-mediated cytokine downregulation and immune suppression. This immunological theme is continued by Clark et al. on the development of Tetrahymena thermophila as a delivery system for recombinant vaccines against parasitic protozoans such as the common fish ciliate Ichthyophthirius multifiliis. Cell lines of T. thermophila expressing T. multifiliis genes under the control of a cadmium-inducible metallothionein gene promoter confer strong protection against a lethal parasite challenge. This protection does not appear to be linked with cadmium being carried over with the vaccine, as fish injected with $T$. thermophilia and cultured in the presence/absence of cadmium, show no significant differences in parasite survival or loading following direct exposure to T. multifiliis.

The final paper by Jobling \& Tyler demonstrates that parasites and pollutants cause endocrine disruption in wild freshwater fish. Except for the tapeworm Ligula intestinalis and two microsporidian protozoans, Pleisistophora mirandellae and Myxobolus sp., relatively few parasites are known to affect the endocrine system of fish and cause reproductive and/or developmental dysfunction. Interactions between the immune and endocrine systems in fish suggest that endocrine disruption, immunotoxicity and hence susceptibility to infection/disease do occur simultaneously in many environments where fish are challenged with pollutants, parasites and other environmental stressors. This is a complex field and much work needs to be done. Nevertheless the recurring themes of the papers in this volume do indicate that, using multidisciplinary approaches, much progress has been and will continue to be made on monitoring the effects of pollution on aquatic hosts and their parasite communities, which in turn will undoubtedly contribute to our understanding of ecosystem functioning both in the mid and long term.

The meeting took place in September 2002 at the Linnean Society of London, Burlington House, Piccadilly, which is now an established venue for the BSP Autumn Symposium. We extend our grateful thanks to Dr John Marsden, Executive Secretary of the Linnean Society, and his staff, especially Janet Ashdown, for their outstanding support and hospitality. We are most grateful to all presenters and coauthors for their contributions to the meeting and to this volume and of course to Les Chappell for his efforts in coordinating the proceedings. Six posters were also presented at the meeting and these include: 'The impact of cadmium on Dactylogyrus extensus', P. Carter et al.; 'The impact of cadmium on Gyrodactylus turnbulli populations on Poecilia reticulata', P. Carter et al.; 'Is there a relationship between the level of imposex in Nucella lapillus populations and the viability of their Parorchis acanthus (Digenea: Philopthalmidae) parasites?', S. Bennett et al.; 'Intersexuality in an estuarine amphipod: pollution, parasitism or both?', A. T. Ford et al.; 'Lernaea and Lamproglena as possible indicators of environmental deterioration in a river in South Africa', A. Avenant-Oldewage; 'The role of fish macroparasites as indicators of pollution', S. Laimgruber et al. Finally, Aquaculture Vaccines Ltd and Cambridge University Press are gratefully acknowledged for providing financial support for the meeting and we sincerely hope that scientists working in the fields of parasitology, ecology, ecotoxicology, 
fisheries and aquaculture will be stimulated to fill the gaps in our knowledge of parasitism and environmental pollution.

\section{REFERENCES}

CHUBB, J. C. (1979). Seasonal occurrence of helminths in freshwater fishes. Part II. Trematoda. Advances in Parasitology 17, 141-313.

CHUBв, J. C. (1980). Seasonal occurrence of helminths in freshwater fishes. Part III. Larval cestoda and nematoda. Advances in Parasitology 18, 1-120.
KENNEDY, C. R. (1997). Freshwater fish parasites and environmental quality: an overview and caution. Parassitologia 39, 249-254.

LAFFERTY, K. D. (1997). Environmental parasitology: what can parasites tell us about human impact on the environment? Parasitology Today 13, 251-255.

MaCKenZie, K., Williams, H. H., Williams, B., McVicaR, A. H. \& SIDDALL, R. (1995). Parasites as indicators of water quality and the potential use of helminth transmission in marine pollution studies. Advances in Parasitology 35, 85-144.

POULIN, R. (1992). Toxic pollution and parasitism in freshwater fish. Parasitology Today 8, 58-61. 